\title{
Perception costs of reproduction can magnify sexual selection
}

To the Editor - In a recent issue of Nature Ecology and Evolution, Harvanek et al. show that male fruit flies (Drosophila melanogaster) experience key survival and physiological costs as a sole consequence of perceiving female pheromones ${ }^{1,2}$. This is an outstanding example of how sensory perception can drastically modulate ageing through different pathways ${ }^{1,3-5}$, presumably by engaging physiological responses evolved to deal with environmental challenges that, in nature, would normally be associated with the perceived stimuli. Crucially, Harvanek et al. show that perception costs of reproduction by exposure to sexual pheromones mostly disappear when male flies have access to regular matings, suggesting that perception of sexual pheromones triggers physiological changes that only result in accelerated ageing when uncoupled with the mating expectations it generates ${ }^{1,2}$. Here, we suggest that such perception costs can magnify sexual selection.

Briefly, if accelerated ageing results from a decoupling between perceived and realized mating opportunities, it follows that low-condition males in a group are open to pay an additional yet previously unrecognized long-term fitness cost. As recognized since Darwin, low-condition males tend to have low fitness because they fare worse than average at intra- and intersexual competition ${ }^{6}$. Harvanek et al. show that low-quality males can suffer extra costs from accelerated ageing because a failure to mate regularly will decouple perception of mating opportunities from mating itself, with concomitant fitness costs. In theory, the importance of such fitness costs will depend on population demography and age-dependent mortality effects of perception costs ${ }^{7}$. In D. melanogaster, such extra costs are realized from early on and can be substantial (15-20\% decrease in average lifespan ${ }^{1,2}$ ), potentially signifying a drastic increase in male variance in lifetime reproductive success.

Sexual selection is fuelled by withinsex variance in reproductive success (that is, the opportunity for selection $)^{8}$, so that the higher the difference between the reproductive success of individuals within each sex in a population, the stronger the a

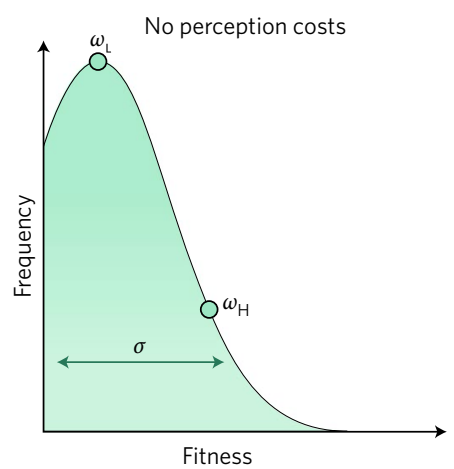

b

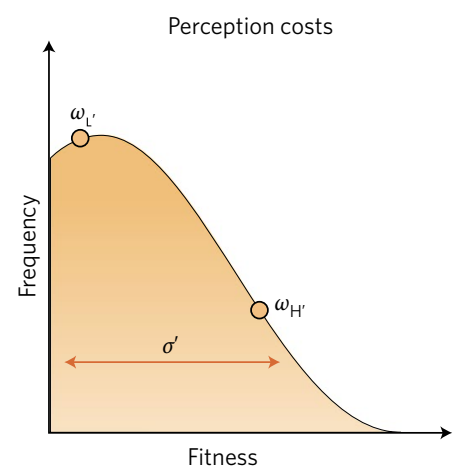

Fig. 1 | How perception costs can magnify sexual selection. a,b, Hypothetical fitness distributions of males in two populations under sexual selection (that is, non-random mating) and no perception costs of reproduction (a) or perception costs of reproduction (b). Perception costs of reproduction are expected to affect low-quality males by accelerating their ageing, hence lowering their fitness (for example, lifetime reproductive success). Assuming overall reproductive output is fixed (for example, fixed number of matings/offspring produced by females), the loss in fitness by low-quality males $\left(\omega_{\mathrm{L}}>\omega_{\mathrm{L}^{\prime}}\right)$ will be capitalized by high-quality males $\left(\omega_{\mathrm{H}}<\omega_{\mathrm{H}^{\prime}}\right)$, increasing the opportunity for selection and hence the upper limit for the action of sexual selection. $\omega_{\mathrm{L}} / \omega_{\mathrm{H}}$ : fitness of low-quality/high-quality male (without perception costs); $\omega_{\mathrm{L}} / \omega_{\mathrm{H}}$ : fitness of low-quality/high-quality male (with perception costs); $\sigma / \sigma^{\prime}$ : variation in fitness without/with perception costs.

action of sexual selection on associated phenotypic differences. As a consequence, perception costs of reproduction will theoretically increase the opportunity for selection (Fig. 1). In turn, this will tend to promote male adaptations that make high-quality males more successful at monopolizing matings, potentially increasing ageing due to perception costs in low-quality males in a positive feedback loop on male fitness variance that will act to strengthen sexual selection.

Correct parameterization of the intensity of sexual selection is pivotal to allow precise quantifications of selection across different mating systems. However, perception costs of reproduction are inherently long term and will not be properly detected by empirical studies that estimate selection based on short-term fitness measures of single cohorts ${ }^{8}$, particularly given that positive feedbacks usually result in nonlinear responses. At a more conceptual level, understanding what factors modulate sexual selection is equally important. For example, sexual selection in males has been suggested to ameliorate or even balance the twofold costs of sex to females via the purging of deleterious mutations ${ }^{9}$, but whether selection on males is strong enough for the maintenance of sex remains an open question ${ }^{10}$. Given that the underlying mechanisms described so far appear to be highly conserved ${ }^{4,5}$, we contend that perception costs of reproduction could play an important yet previously unrecognized role in the evolution of male and female adaptations and life histories. We outline two avenues for future research: (1) in model organisms (for example, D. melanogaster), manipulating perception costs in males of varying quality and subsequently testing whether this results in an increase or decrease of male fitness variance; and (2) comparing short-term (that is, no perception costs) versus long-term estimations of male fitness variance in single cohorts via meta-analysis or ad hoc behavioural experiments.

Pau Carazo ${ }^{1 \star}$, Roberto García-Roa', Zahida Sultanova ${ }^{1}$ and Manuel Serra ${ }^{2}$ 
${ }^{1}$ Ethology, Behaviour and Evolution Lab, Cavanilles Institute of Biodiversity and Evolutionary Biology, University of Valencia, Valencia, C.P. 46980, Spain. ${ }^{2}$ Evolutionary Ecology Lab, Cavanilles Institute of Biodiversity and Evolutionary Biology, University of Valencia, Valencia, C.P. 46980, Spain.

*e-mail:pau.carazo@uv.es

Published online: 04 September 2017

DOI: 10.1038/s41559-017-0312-6
References

1. Gendron, C. M. et al. Science 343, 544-548 (2014).

2. Harvanek, Z. M. et al. Nat. Ecol. Evol. 1, 0152 (2017).

3. Apfeld, J. \& Kenyon, C. Nature 402, 804-809 (1999).

4. Libert, S. et al. Science 315, 1133-1137 (2007).

5. Linford, N. J., Kuo, T. H., Chan, T. P. \& Pletcher, S. D. Annu. Rev. Cell Dev. Biol. 27, 759-785 (2011).

6. Darwin, C. The Descent of Man and Selection in Relation to Sex (John Murray, London, 1871).

7. Tatar, M. \& Promislow, D. E. L. Evolution 51, 1323-1326 (1997).

8. Schuster, S. M. \& Wade, M. J. Mating Systems and Strategies (Princeton-MBE, Princeton, 2003).
9. Agrawal, A. F. Nature 411, 692-695 (2001).

10. Prokop, Z. M. et al. Evolution 71, 650-661 (2017).

Author contributions

P.C. conceived the idea. P.C., R.G.-R., Z.S. and M.S.

further developed the theory. P.C. wrote the paper with contributions from R.G.-R., Z.S. and M.S.

Competing interests

The authors declare no competing financial interests. 